\title{
Context: the grey matter of cancer
}

As the ESMO conference approaches, we pause to reflect on the latest progress, key challenges, and future directions in clinical oncology. What has the plethora of research and clinical trial data from thousands of publications taught us? That context is paramount. Our ability to understand evolutionary cancer biology in exquisite detail is now possible. Yet, our efforts to translate these advances into the clinic and make tangible differences to patients continue to disappoint. Biology is undoubtedly a highly complex, adaptive, multi-networked process; thus, so is cancer. Are we even in agreement on how we define cancer? I'd argue no. It is an unpredictable moving target that disguises and reinvents itself on a constant basis. Since cancer is a non-organ or systems-based disease, our starting point is already hindered. To begin to understand resistance - the Holy Grail - we should take a critical look at some of our definitions. Our knowledge is extrapolated from diverse timeframes, clinical indicators, and treatment response observations. The crux is to provide, in a contextual manner, a means of understanding this disease in a world that lacks well-defined and, importantly, context-adaptable descriptors. This issue is compounded further by cost contraints, infrastructural issues and the quest to mine and structure the petabytes of human data, which we are drowning in.

While sobering, we can take some solace in scientific progress. We are now beginning to understand and witness the significance of tumour-cell intrinsic and extrinsic co-dependencies. The surrounding 'nonmalignant' tissue can reprogramme or establish the framework for how cancer cells respond and grow - these symbiotic interactions are intercollaborative, co-dependent networks that feed off each other. Moreover, we have started to appreciate that certain oncogenic alterations cluster or occur together, whereas others are mutually exclusive. These findings suggest that mutations are conditionally selected, and confirm that tumour evolution is not random. Using genomic-based algorithmic approaches, researchers have developed methodological frameworks to study each tumour as a system of cooperating events to generate maps of oncogenic dependencies. Yet, how much of context is captured in routine experiments, or in our ability to translate preclinical findings to the bedside? Surprisingly little. So, what is the answer?

Our past reductionist approaches have led us to miss the fuller picture, look in the wrong place, or worse still, not even question if we have the right starting point. We define and categorize similarities and differences of cancer and the 'normal' tissue as a means of target identification and treatment development. While this is logical and empirical, does biology work this way? No. We need to ask ourselves, therefore, 'what would the disease do'? Context is underpinned in every 'observation', 'intepretation', and resultant 'decision' that biological networks make. In everyday life, several factors are considered simultaneously to influence our decisions: one small deviation can result in an entirely different decision or outcome. Cancer is no different, and does this autonomously - so we need to understand this better.

We define genes as tumour suppressors or oncogenes, drivers or passengers, with little consideration that they can be both in the same tumour depending on context. We often see what we want to see, and disregard what does not conveniently tally with our simplistic definitions. Even our grant-writing and funding processes are typically based on us knowing the answers before we've asked the questions. The creative freedom in scientific discovery has suffered in recent years, largely because of career and publication pressures, and economic competition. The danger is that this might result in suboptimal patient care. The irony is that, in some respects, cancer shows a similar trend; for instance, in advanced-stage disease some of the suppressive mechanisms and immune equilibrium checkpoints become more restricted, which might paradoxically provide opportune avenues to circumvent these barriers, providing the disease context is understood more fully at an earlier stage in its timecourse. With improvements in animal models and our ability to subtly regulate genetic and epigenetic modifications in the lab, and correctly interpret the findings, we can hopefully begin to make a difference for patients.

The next challenge is how we define these nuances in the results achieved in our modern clinical trials. This is not going to be easy because our ever-increasing list of drugs and how such combinations might be assessed clinically will not be possible in practice or optimally related to the 'real-world' patient population. Trials should be context-specific and representative of the patients that the drugs will be used in outside of trials. This is where Nature Reviews Clinical Oncology has a key role and responsibility in interpreting the data and teasing out the signal from the noise in the vast and confusing literature. Context is the grey (not the black or white) matter of cancer. I hope the cancer community at large does not overlook context - as this should underpin everything we encompass when studying this disease. I say farewell as Chief Editor of this journal, but know context will be honoured in the future. 